\title{
A Real-Time Performance Evaluation of Tightly Coupled LTE Wi-Fi Radio Access Networks
}

\author{
Thomas Valerrian Pasca S, Sumanta Patro, Bheemarjuna Reddy Tamma, and Antony Franklin A \\ Department of Computer Science and Engineering, IIT Hyderabad \\ Email: [cs13p1002, cs15mtech01005, tbr, antony.franklin]@iith.ac.in
}

\begin{abstract}
A tight coupling of LTE and Wi-Fi interfaces can be achieved by integrating them at their radio protocol stacks. LTE and Wi-Fi radio level integration with IPSec tunnel (LWIP) was introduced by 3GPP as part of Rel-13. This tighter level of interworking replaces the traditional way of cellular-Wi-Fi interworking through a packet gateway and it can react to the dynamic changes in the wireless link quality. In this paper, we present a variant of LWIP prototype that works with commercial UE (Nexus 5). The developed LWIP prototype uses OpenAirInterface (OAI) for LTE network and Cisco Access Point (AP) as WiFi AP. We also present the design and implementation of LWIP prototype and interesting results for tight interworking of LTE and Wi-Fi at IP level. We have evaluated the LWIP performance with different Link Aggregation Strategies (LAS) using both UDP and TCP. We have observed that, in a highly loaded Wi-Fi channel, when LWIP employs Wi-Fi only in Downlink (WoD) LAS, then sum of individual TCP flow throughput has improved by $\mathbf{2 8 \%}$ as compared to LWIP operating with Flow Split (FS) LAS. We have enumerated the challenges which has to be addressed in LWIP to reap the maximum benefits.
\end{abstract}

\section{INTRODUCTION}

The Cisco VNI forecast elucidates that the data traffic generated by mobile devices is growing at an exponential rate, than it could ever imagine. As per [1], monthly mobile data traffic will reach 49 exabytes by 2021 , up from 7.2 exabytes per month in 2016. Operators look for a best solution to cater this ever increasing demand. LTE-Wi-Fi interworking is one such technology which can serve this high data requirement. The problem which exists with LTE-Wi-Fi interworking is their underlying interworking architecture. The interworking from Rel-8 to Rel-11 is completely realized through offloading (i.e., moving a flow completely from LTE interface to Wi-Fi interface and vice-versa). Such flow offloading requires a change in the flow route from the cellular core network to the Wi-Fi network. Frequent flow routing across LTE and WiFi networks at Evolved Packet Core (EPC) is inefficient for dense small cell deployments. To address this problem, and to serve delay bounded services and to increase the flexibility in offloading, there is a necessity for these two radios to work much closer. Hence, LTE-Wi-Fi radio level integration with IPSec Tunnel (LWIP) has evolved realizing a tighter integration by associating a Wi-Fi radio next to LTE radio which facilitates an enhanced control over both the radios. LWIP realizes the interworking benefit at link level for better quality of service with seamless flow mobility across LTE and Wi-Fi interfaces. LWIP has the following advantages:
- The LTE core network is unaware of the existence of a Wi-Fi interface.

- LTE acts as licensed anchor point for communication.

- Radio level interworking allows effective utilization of LTE and Wi-Fi links.

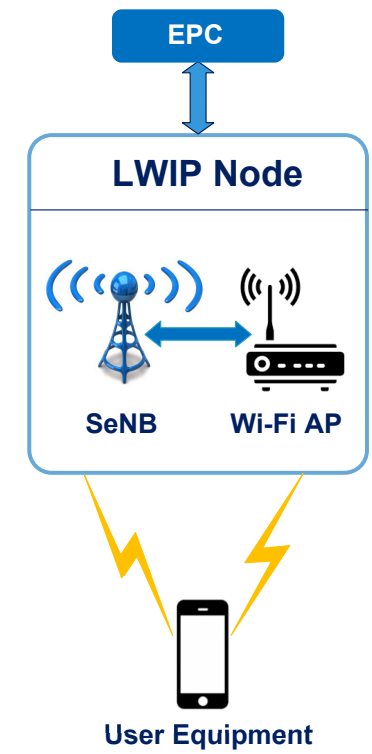

Fig. 1: Tightly coupled LTE Wi-Fi radio access networks.

LWIP is emerging as a competing technology to LTE-U [2] which supports LTE like transmission in unlicensed band. This paper concentrates on design, implementation, and performance of LWIP technology. In principle, LWIP could be realized in two ways, (1) Collocated LWIP, and (2) Non-collocated LWIP. A collocated LWIP node includes an LTE Small cell evolved-NodeB ( SeNB) and Wi-Fi AP are colocated in same device and tightly integrated at Radio Access Network (RAN) level which are driven by finer control decision with combined intelligence. Unlike collocated LWIP, a non-collocated LWIP requires an intelligent decision making in steering data because of heterogeneity in the round trip delay between LTE and Wi-Fi links. In this paper, we describe our developed noncollocated LWIP testbed, that has been developed using opensource platforms such as OpenAirInterface [3] for LTE module and Cisco AP/Hostapd [4] for Wi-Fi module.

The rest of the paper is organized as follows: Section II discusses LWIP architecture and its benefits. We then present in detail the implementation of LWIP testbed using OAI 
in Section III. In Section IV, LWIP testbed performance is evaluated by employing different Link Aggregation Strategies (LAS). Finally, in Section V, we present concluding remarks.

\section{ARChITECTURE PROPOSAL AND STANDARDS}

Third-Generation Partnership Project (3GPP) has shed light on standardization of data offloading to WLAN from Release 8 onwards. They include, Rel-8: PMIP based mobility and Access Network Discovery and Selection Function (ANDSF), Rel-9: enhanced ANDSF (eANDSF), Rel-10: IP Flow mobility (IFOM), Rel-11: location based selection of gateway for WLAN, and Rel-12: WLAN network selection, Multiple PDN connections, and IP preservation. All these schemes focus on realizing LTE-Wi-Fi interworking through Evolved Packet Core (EPC) core (such as, at Serving Gateway (S-GW) and Packet Gateway (P-GW)). The granularity of offloading in these schemes is at flow level i.e., moving a flow completely from one interface to another interface. These gateway based solutions are not quick in the case of user mobility, and dynamic channel variations (e.g., shadowing and fading). To quash from this inefficiency in regulating traffic flows across LTE and Wi-Fi networks, finer control over interfaces is required, it could be achieved only if the decision making entity for offloading is adjacent to RAN part of LTE and Wi-Fi networks. This requirement has impelled the decision making entity all the way from the EPC to SeNB, which ensures a tight coupling between LTE and Wi-Fi RANs.

Integration of LTE and Wi-Fi RANs can be comprehended at different layers of protocol stack. 3GPP has standardized aggregation of Wi-Fi with eNB at PDCP layer [5] and this finer level of aggregation is named as LTE-Wi-Fi Aggregation (LWA). It uses reordering procedure followed in case of Dual Connectivity (DC) in LTE to ensure in-sequence delivery of packets to UE. In [6], authors have studied the performance of TCP in LWA using a real time testbed and observed that PDCP reordering timer has an adverse impact on TCP. A new architecture, coined as LWIR [7], for efficient LTE and WLAN aggregation at the RLC layer of LTE eNodeB has been proposed. In LWIR architecture, the packet from cellular core network is steered at RLC into LTE or Wi-Fi MAC queues. In this architecture, RLC takes care of reordering and retransmission across LTE and Wi-Fi links. Similarly, A tighter level of integration at IP layer, known as LWIP [8] and it is standardised by 3GPP. Figure 1 shows the tightly coupled LTE-Wi-Fi radio access network architecture. LWIP architecture implements an IPSec tunnel between LTE eNB and UE through Wi-Fi link. Figure 2 shows the protocol implementation of LWIP Node as proposed by 3GPP.

Both LWA and LWIR architectures need modification at the protocol stack of the UE. Among tightly coupled architectures, only LWIP architecture supports the existing commercial UEs to work readily with LWIP node (aggregated LTE SeNB and Wi-Fi AP). Hence, we have focused on developing interworking at IP level and developed the LWIP prototype.

We have implemented a complementing and more tighter level integration at IP layer to perform traffic steering at the

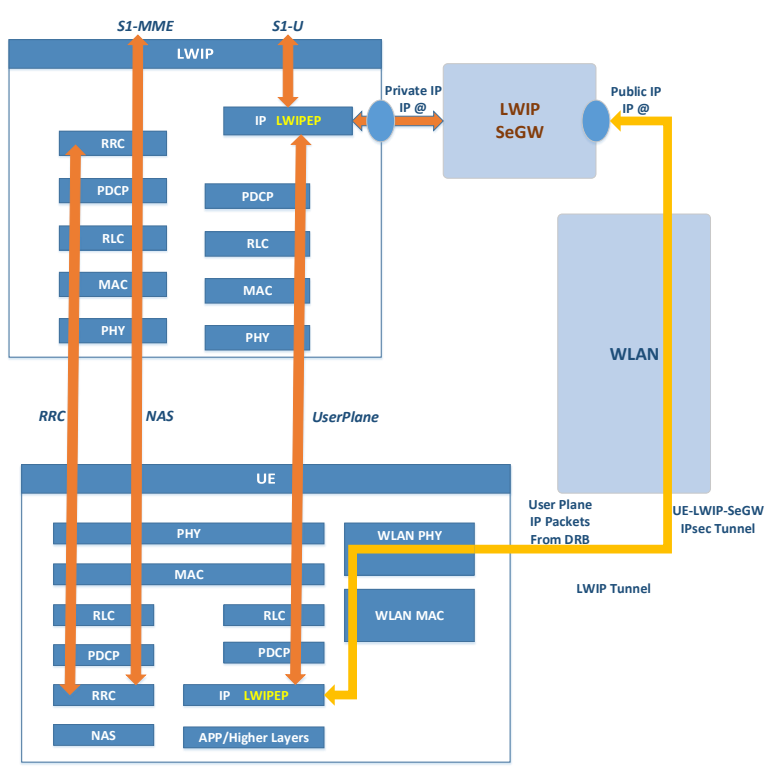

Fig. 2: Protocol stack of LWIP proposed by 3GPP [8].

granularity of bearer level, flow level, and packet level. This traffic steering is done above the PDCP layer of LTE and the LLC layer of Wi-Fi in their respective protocol stacks. Based on the traffic steering mechanism, the traffic steering layer decides which packets/flows/bearers to be transmitted over LTE and Wi-Fi and sends them over the corresponding radio interface. LWIP is realized by introducing a Link Aggregation Layer (LAL) in the protocol stack of LWIP node. LAL does not add any new header to the IP data packets received from EPC via S1-U interface. Packets going through LTE and Wi-Fi interfaces follow regular packet forwarding procedures at their protocol stacks and get delivered directly to IP layer.

LWIP is leveraged by its ease of implementation to achieve the aggregation benefit. Also, LAL supports collecting various network parameters and actively participates in intelligent decision making for steering IP traffic across LTE and Wi-Fi interfaces in the downlink. It is notable that UE does not require any modification to the protocol of UE.

\section{Testbed Setup}

LWIP testbed is setup using OpenAirInterface (OAI) as LTE network and Cisco access point as Wi-Fi AP. To the best of our knowledge, this is the first ever reported performance evaluation of LWIP prototype.

\section{A. LTE Testbed using OpenAirInterface}

OAI [9] is a complete implementation of 4G-LTE (Rel10), it includes OpenAirInterface User Equipment (OAI-UE), OpenAirInterface eNodeB (OAI-eNB), and OpenAirInterface Core Network (OAI-CN). OAI-eNB works steadily with commercial UE such as Nexus 5, Samsung Galaxy S5, iPhone 5s and others.

OAI-eNB contains MAC, RLC, PDCP, and RRC layers according to 3GPP standard. It also supports eMBMS services $(\mathrm{MCH}, \mathrm{MCCH}, \mathrm{MTCH})$. It has regular RRC measurement 


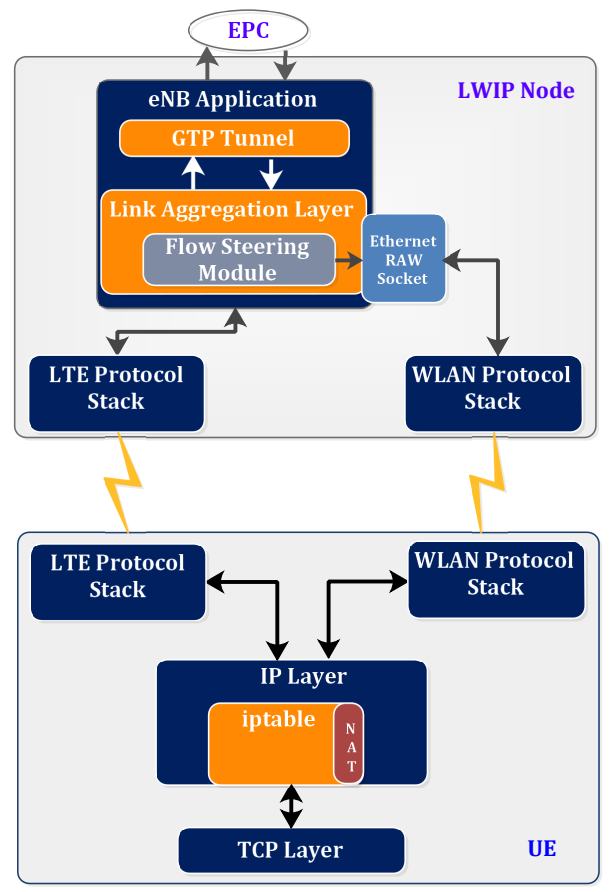

Fig. 3: Protocol implementation structure of LWIP prototype.

with measurement gap. Communication of S1-AP and GTP-U interfaces with core network occur according to 3GPP.

OAI-CN has EPC components which comply with 3GPP standards up to Rel-10. It includes Serving Gateway (S-GW), Packet Data Network Gateway (P-GW), Mobility Management Entity (MME), Home Subscriber Server (HSS) and NonAccess Stratum (NAS).

LTE hardware ExpressMIMO2 (ExMIMO2) PCI express boards are used as RF frontend in the testbed setup. ExMIMO2 boards belong to the class of Software Defined Radio (SDR), that can work upto $80 \mathrm{MHz}$ of carrier aggregation [10].

\section{B. Realization of LWIP Testbed using OAI}

Figure 3 illustrates the protocol implementation structure of LWIP prototype. We have implemented the LWIP architecture proposed in [11], which includes a minor modification to 3GPP LWIP architecture. The main difference between 3GPP and implemented architecture is, there is no IPSec tunnel to deliver the packet to destined UE. Security aspects of LWIP is not our primary focus in this paper, however, we have detailed the security procedures of proposed architecture in [12]. The actual destination IP address of the packet is changed from LTE IP address to Wi-Fi IP address at LWIP node and at UE, the destination IP address is changed back to LTE IP address. Our LWIP testbed setup is shown in Figure 4, which is demonstrated in [13]. The following challenges are addressed for realizing a fully functional LWIP testbed.

- A socket connection with the destination should be done with LTE interface IP at UE - In Android operating system (OS), when Wi-Fi radio is enabled, it gets the highest priority over all available radio interfaces. This priority issue has to be solved because the connection is LTE anchored. With a stringent motive of making existing UE to work, we have developed an Android application which changes priority for set of flows to use LTE interface for communication.

- The Wi-Fi interface details of UE has to be informed to LTE SeNB - The re-routing source should be aware of the destination interface for enabling re-routing. In our LWIP testbed, the information of UE's Wi-Fi IP address is made known to LWIP node.

- A packet from LTE core network has to be re-routed to Wi-Fi network - This action mangles the packet headers to achieve successful routing between LTE and Wi-Fi networks, also it involves recomputing the header checksum of appropriate layers in order to avoid the packet drop at the destination. In our LWIP testbed, the actual packet destination address is changed by LWIP node in order to deliver the packet over Wi-Fi interface. When the destination IP gets changed, all the higher layer headers are recomputed for the mangled packet.

- An unmodified connection between LTE-SeNB and UE through Wi-Fi interface has to be maintained Packet received at the destination (UE) should be able to deliver the packet to the socket to which it is bounded to. If it is not transformed, packet gets lost. In our LWIP testbed, the packet on reaching the UE with Wi-Fi IP address is changed back to LTE IP address with the help of iptable rules, thereby managing the connection alive even through Wi-Fi interface.

LWIP testbed configurations are shown in Tables I and II. In our testbed, the UE, Nexus 5, is downloading a file from the remote server using LWIP. Both interfaces are enabled through an Android application. We have enhanced the open-source Android application HIPRIKeeper [14] which can enable both LTE and Wi-Fi interfaces at the same time to test the LWIP operation. State of a flow is unalterably maintained by inserting flow rules in iptable of UE. This iptable rule makes the packet look as if it is unaltered to the destination socket and seamless. Hence, it enables aggregation of LTE and Wi-Fi networks to work uninterruptedly.

TABLE I: EXPERIMENTAL PARAMETERS

\begin{tabular}{|c|c|}
\hline Parameter & Value \\
\hline LTE eNB Bandwidth & $5 \mathrm{MHz}$ \\
\hline Number of Resource Blocks & 25 \\
\hline Tx Power & $20 \mathrm{dBm}$ \\
\hline Scheduler & Round Robin \\
\hline Wi-Fi Frequency, Bandwidth & $2.4 \mathrm{GHz}, 20 \mathrm{MHz}$ \\
\hline Wi-Fi Standard & IEEE $802.11 \mathrm{~b}$ and g \\
\hline
\end{tabular}

\section{Performance Evaluation}

In this section, we evaluate the developed LWIP prototype using different LAS which are detailed in the following subsections. The performance of LWIP is studied using following experiments.

1) iPerf [15] using UDP - LTE, Wi-Fi, and LWIP.

2) TCP performance - When different LAS are employed. 


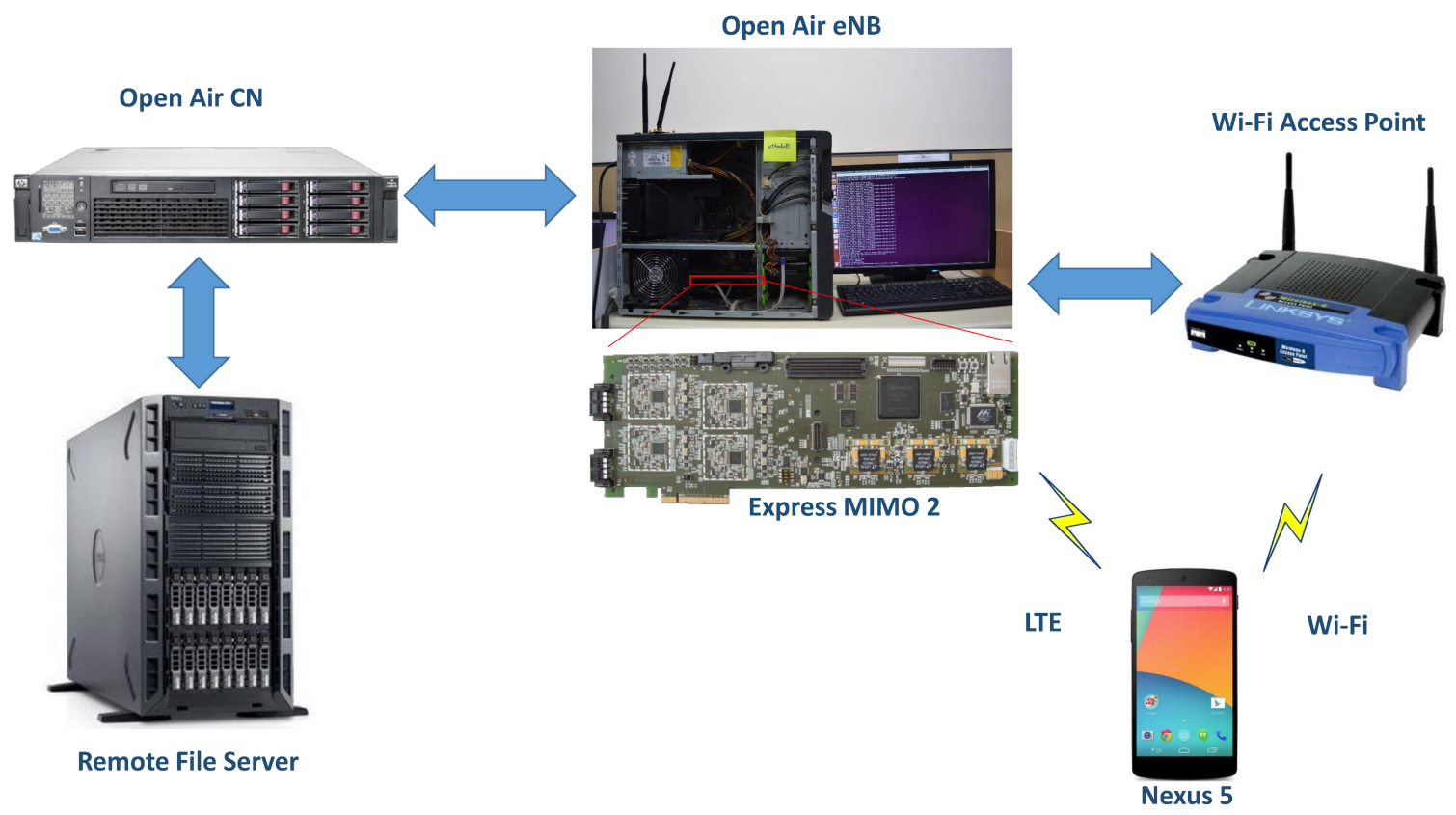

Fig. 4: LWIP Testbed Setup.

TABLE II: TESTBED SETUP CONFIGURATION

\begin{tabular}{|c|c|}
\hline Parameter & Value \\
\hline OAI LTE eNB & ExMIMO2, Intel Xeon 8 core, \\
Hardware Config & 12GB DDR, Gigabit Ethernet 1 Gb/s \\
\hline OAI LTE eNB & Ubuntu 14.04, Low Latency Kernel 3.19 \\
Software Config & \\
\hline OAI EPC & Intel Xeon Server 24 core, 64GB DDR, \\
Hardware Config & Gigabit Ethernet 10 Gb/s \\
\hline OAI EPC & Ubuntu 14.04, Kernel 3.19 generic \\
Software Config & Intel Xeon 8 core, 32GB DDR, \\
\hline Remote Server & Gigabit Ethernet 1 Gb/s \\
Harware Config & Ubuntu 14.04, Kernel 3.2 \\
\hline Remote Server & Apache 2 Webserver, TCP - High Speed \\
Software Config & Nexus 5 - hammerhead, Android 4.4.4 (KitKat) \\
\hline User Equipment
\end{tabular}

\section{A. UDP Test using iPerf}

UDP iPerf test is conducted from a server in local network to the UE. In this experiment, LWIP is setup using IEEE $802.11 \mathrm{~b}$ and $\mathrm{g}$ in different tests. The experiment includes evaluating the performance of using LTE, Wi-Fi and LWIP. In case of LWIP, the LWIP node steers a set of input packets through LTE and Wi-Fi based on the ratio of their link rates. Figures 5 and 6 illustrate the throughput observed during the iPerf test in our experimental LWIP testbed. In Figure 5, where IEEE $802.11 \mathrm{~b}$ is used as Wi-Fi AP, the LWIP performance is nearly equal to sum of combined throughput of LTE and Wi-Fi links, since the link rates of LTE and IEEE $802.11 \mathrm{~b}$ are comparable. Whereas in case of IEEE $802.11 \mathrm{~g}$, LWIP is able to achieve throughput close to the throughput of using strictly Wi-Fi. This is because we have split the downlink traffic across LTE and Wi-Fi links at LWIP node in a fixed ratio corresponding to LTE and WiFi link rates. But the link capacity varies dynamically due to variations in the channel. Hence, the throughput observed in LWIP is not equal to sum of throughput observed in LTE and Wi-Fi links. This puts forth the need for efficient traffic steering algorithm which can yield better benefits. We have not addressed this problem here since it is beyond the scope of this paper. Figure 7 shows CDF of observed jitter during the experiment, LWIP jitter is higher than using strictly Wi-Fi but it is lesser than using strictly LTE. In summary, finer level of integration has improved the throughput when link rates are comparable and demands an efficient steering algorithm if incomparable link speeds are used.

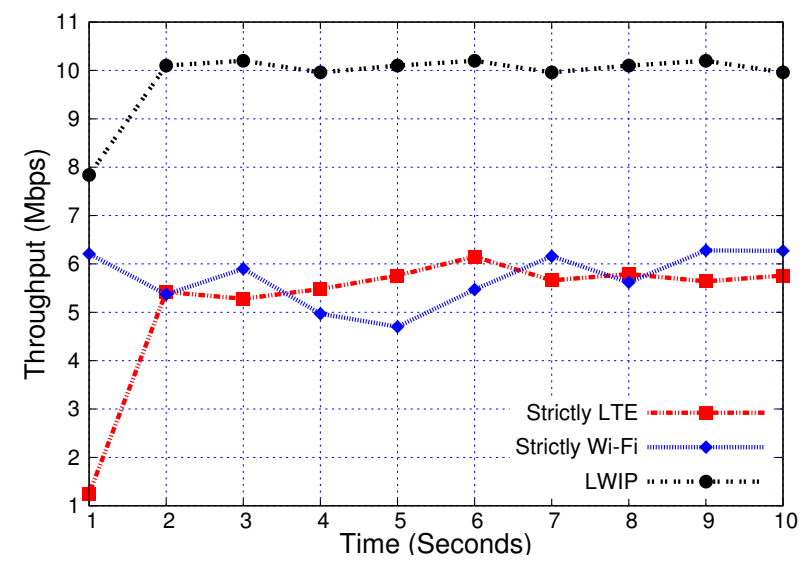

Fig. 5: Throughput in iPerf test using UDP (in downlink - 802.11 b).

\section{B. TCP Experiment in LWIP Testbed}

To observe the performance for different Link Aggregation Strategies, we have conducted a set of experiments by analysing their performance in low and high load scenarios. Wi-Fi AP uses IEEE 802.11 g standard for following experiments. We have developed an Android application, which 


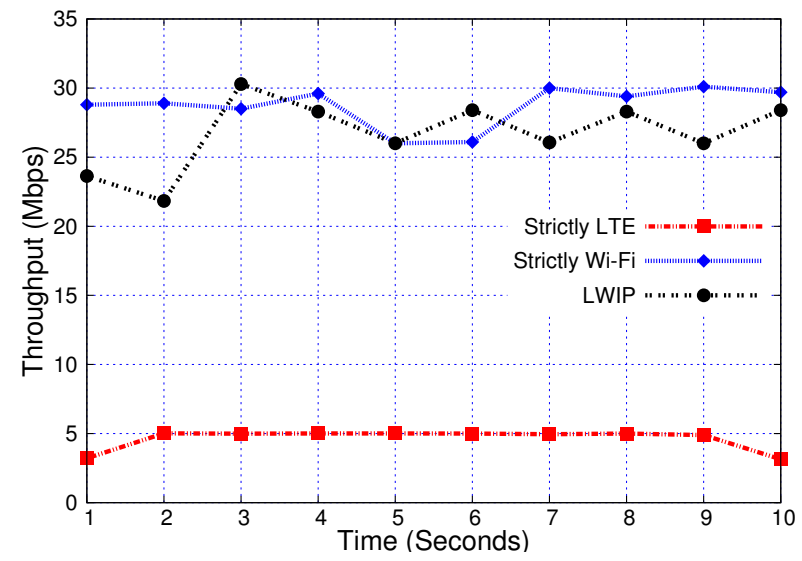

Fig. 6: Throughput in iPerf test using UDP (in downlink - $802.11 \mathrm{~g}$ ).

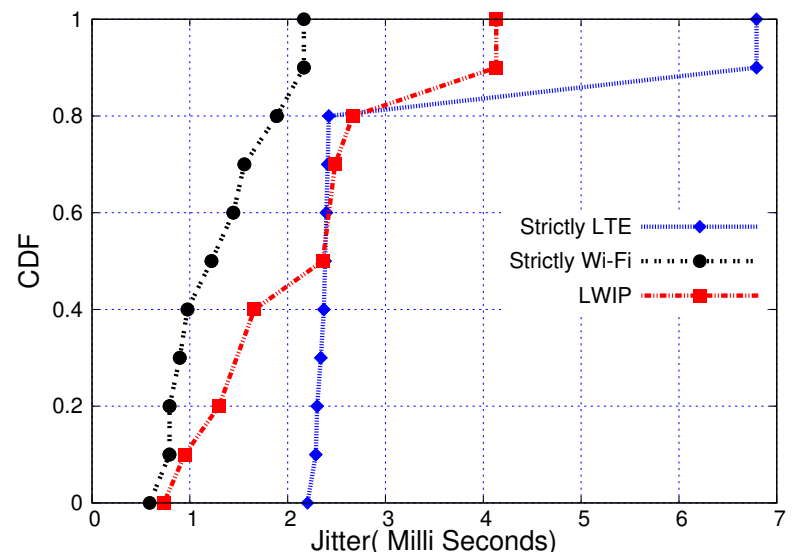

Fig. 7: Jitter CDF for iPerf test.

downloads two files simultaneously from a remote server and measures their throughputs. The UE performance is evaluated using the following LAS,

- LTE NoLAS: Two flows are simultaneously downloaded through LTE.

- Wi-Fi NoLAS: Two flows are simultaneously downloaded through Wi-Fi.

- FS-LAS: Flow split enables one flow to be downloaded through LTE and other through Wi-Fi.

- WoD-LAS: WiFi only in Downlink enables both the flows to use Wi-Fi for downlink and the corresponding TCP ACKs are sent through LTE in uplink.

TCP Experiment 1 - Lightly Loaded Scenario: The setup consists of a UE and a LWIP node with background transmission in Wi-Fi channel (observed channel load is $8 \%$ ). Now, UE downloads two files from the remote server using different LAS. We have downloaded files of different sizes viz., 16 and $32 \mathrm{MB}$. Figure 8 shows the throughput observed in case of different file downloads. It can be observed that FS-LAS has achieved higher throughput, since it effectively aggregates the LTE and Wi-Fi links. Also it is better compared to WoD-LAS because of the type of steering it employs. Figure 9 shows that all the LAS employed utilizes Wi-Fi link at its maximum link rate. Time to download a file using different LAS is shown in Figure 10. Even though the throughput of FS-LAS and WoD-LAS are comparable, the time to download a file through FS-LAS incurs longer time than WoD-LAS because file download through LTE interface incurs longer download time.

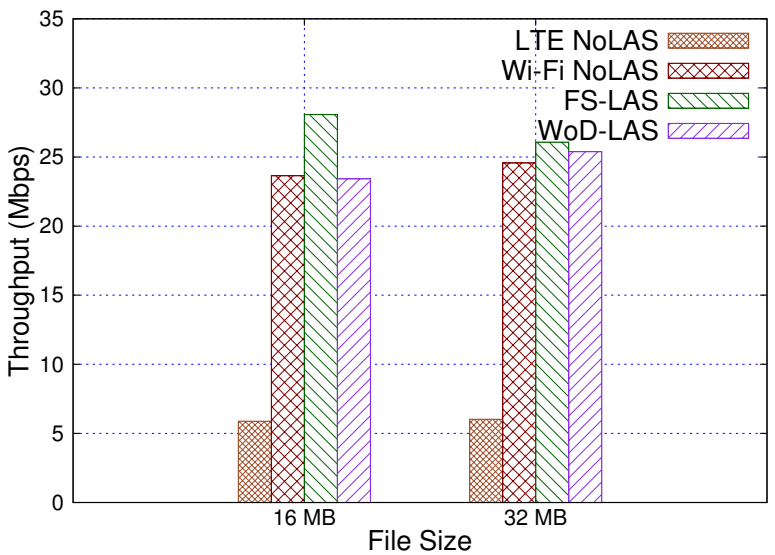

Fig. 8: Overall Throughput observed for $16 \mathrm{MB}$ and $32 \mathrm{MB}$ file sizes with low contention.

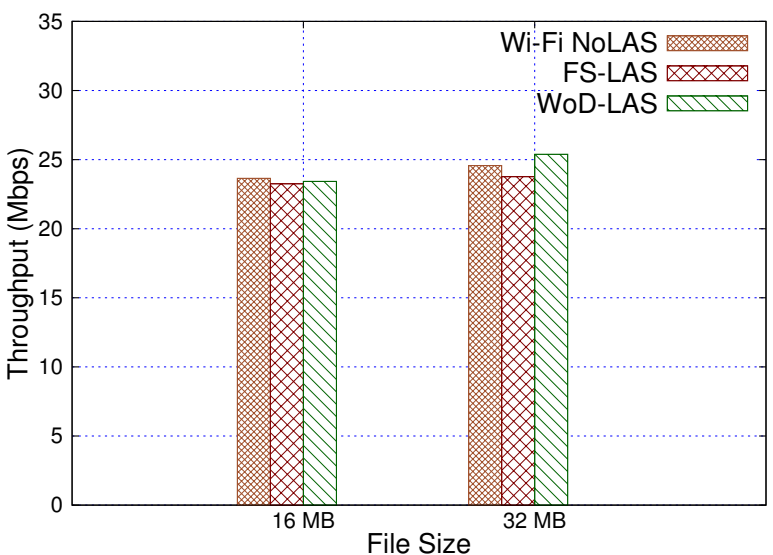

Fig. 9: Throughput of Wi-Fi observed for $16 \mathrm{MB}$ and $32 \mathrm{MB}$ file sizes with low contention.

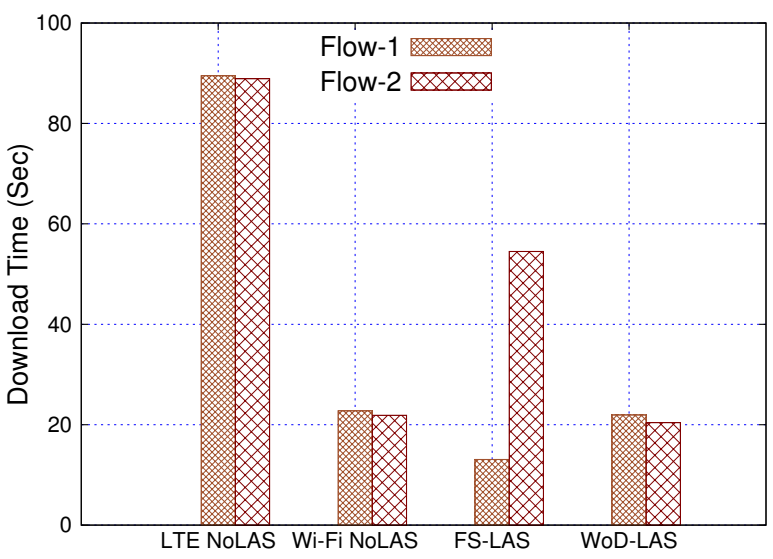

Fig. 10: Time to download a $32 \mathrm{MB}$ file with low contention.

TCP Experiment 2 - Heavily Loaded Scenario: In this setup, for creating a heavily loaded condition, we have used five laptops, each of those streams video at high bit rate (900 kbps UDP stream per device) to an AP operating in the same Wi-Fi channel of LWIP node. This newly introduce load is in addition to existing $8 \%$ background Wi-Fi channel 
load. Now, the LWIP system performance is analyzed using different LAS. Figure 11 shows that with high load, throughput of FS-LAS and WoD-LAS has reduced by $28 \%$ compared to scenario with lower load. Contention in the channel has brought down the throughput of UE. Figure 12 shows that performance of WoD-LAS has improved compared to FS-LAS. In case of FS-LAS, the TCP ACK packets which are generated for the flow through Wi-Fi have to be sent through Wi-Fi link only. Since Wi-Fi contention is high, it brings down the throughput of that flow. This problem is solved when WoD-LAS is employed, as uplink of LTE does not have contention unlike uplink of Wi-Fi, hence it achieves a higher throughput. Figure 13 shows the time to download two files using different LAS, which are $30 \%$ high as compared to low loaded scenario.

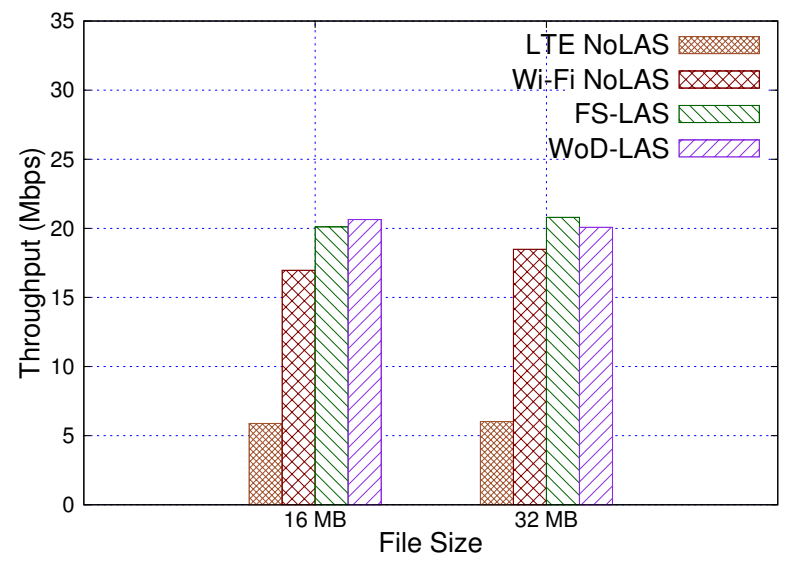

Fig. 11: Overall Throughput observed for $16 \mathrm{MB}$ and $32 \mathrm{MB}$ file sizes with high contention.

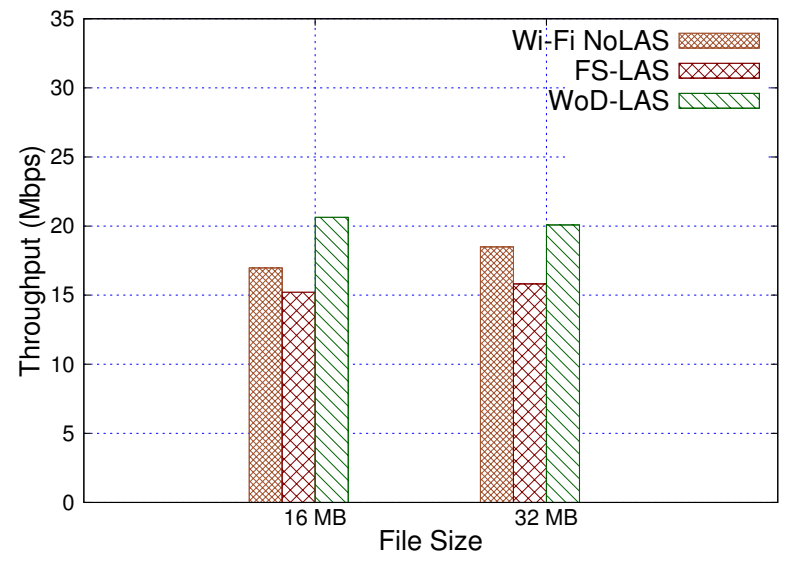

Fig. 12: Throughput of Wi-Fi observed for $16 \mathrm{MB}$ and $32 \mathrm{MB}$ file sizes with high contention.

\section{CONCLUSIONS AND Future WORK}

This paper demonstrates the feasibility of LTE-Wi-Fi radio level integration at IP layer using OAI LTE, Cisco AP, and commercial UE. The developed prototype does not require any modifications to the protocol stack of the UE. We have implemented different link aggregation strategies to study the performance of the LWIP prototype. The experiment results

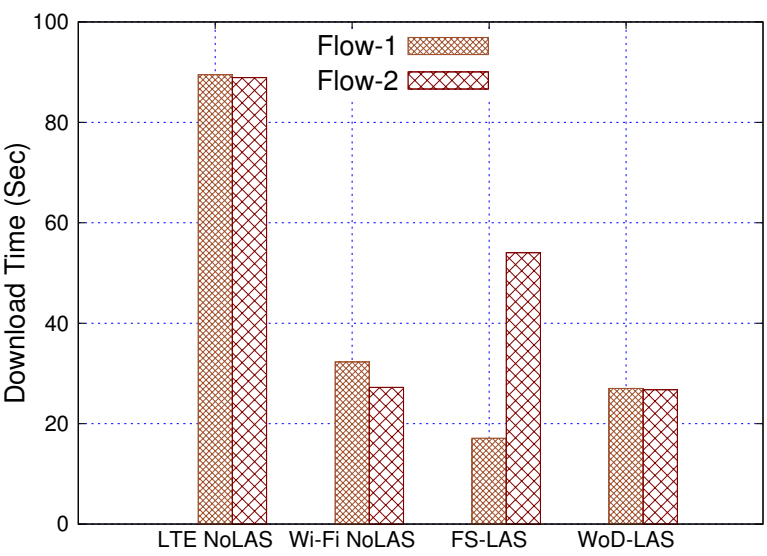

Fig. 13: Time to download a $32 \mathrm{MB}$ file with high contention.

conclude that WoD-LAS has improved sum of flow throughputs by $28 \%$ as compared to FS-LAS, when the contention in Wi-Fi channel is high. Thus enabling WoD-LAS will be the most preferred link aggregation technique. There are numerous research challenges in a practical environment pertaining to real-time LWIP on flow and packet level routing which can be well studied using this testbed. As part of our future work, we will implement efficient packet level steering solutions across LTE and Wi-Fi links to improve TCP performance.

\section{ACKNOWLEDGEMENT}

This work was supported by the project "Converged Cloud Communication Technologies", MeitY, Govt. of India.

\section{REFERENCES}

[1] Mobile Network in 2017. [Online]. Available: https://goo.gl/ylTuVx

[2] B. Chen et al., "Coexistence of lte-laa and wi-fi on $5 \mathrm{ghz}$ with corresponding deployment scenarios: A survey," IEEE Communications Surveys \& Tutorials, vol. 19, no. 1, pp. 7-32, 2017.

[3] OpenAirInterface. [Online]. Available: www.openairinterface.org/

[4] L. Surhone, M. Timpledon, and S. Marseken, Hostapd. VDM, 2010.

[5] 3GPP. (2016) LTE-WLAN Aggregation and RAN Controlled LTEWLAN Interworking (LWA). [Online]. Available: http://www.3gpp.org/ DynaReport/36300.htm

[6] Y. Khadraoui et al., "TCP Performance for Practical Implementation of Very Tight Coupling between LTE and WiFi," in IEEE 84th Vehicular Technology Conference (VTC-Fall), 2016, pp. 1-6.

[7] P. Sharma et al., "LWIR: LTE-WLAN Integration at RLC Layer with Virtual WLAN Scheduler for Efficient Aggregation," in IEEE GLOBECOM, 2016.

[8] 3GPP. LTE/WLAN Radio Level Integration Using IPsec Tunnel (LWIP) encapsulation; Protocol specification. [Online]. Available: http://www.3gpp.org/DynaReport/36361.htm

[9] N. Nikaein et al., "Openairinterface: A flexible platform for $5 \mathrm{G}$ research," ACM SIGCOMM CCR, vol. 44, no. 5, pp. 33-38, 2014.

[10] — "Demo: Openairinterface: an open lte network in a pc," in MOBICOM. ACM, 2014, pp. 305-308.

[11] T. Bheemarjuna Reddy, F. Antony, and S. Thomas. Traffic steering strategies for LWA. [Online]. Available: www.tsdsi.org/standards/swip/ $30 /$

[12] S. Thomas et al., "Architectural Challenges and Solutions for Collocated LWIP - A Network Layer Perspective," in NCC, 2017.

[13] S. Thomas, P. Sumanta, T. Bheemarjuna Reddy, and F. Antony, "Tightly coupled LTE Wi-Fi radio access networks: A demo of LWIP," in Proc. of 9th International Conference on Communication Systems and Networks (COMSNETS). IEEE, 2017, pp. 419-420.

[14] HIPRIKeeper. [Online]. Available: https://goo.gl/jP7bja

[15] D. Jon et al. iPerf. [Online]. Available: https://iperf.fr/ 